\title{
A Review of Fractal Tree Antennas*
}

\author{
Hongming Zang ${ }^{1,2}$, Xiaoying $\mathrm{Zhao}^{2}$, Xiong $\mathrm{Xu}^{1}$,Gonglei Zhang ${ }^{2}$ and Jingjing $\mathrm{Lu}^{2}$ \\ ${ }^{1}$ The State Key Laboratory of Complex Electromagnetic Environmental Effects on Electronics \& Information System, Luoyang, \\ China \\ ${ }^{2}$ School of Electronic \& Information Engineering, Beihang University, Beijing, China \\ zanghongming410@126.com, xyz@buaa.edu.cn, \{xuxiongly \& zglbhq \& chaorenqijinger\}@163.com
}

\begin{abstract}
Fractal tree structures can be utilized to design antennas in order to enhance the performance of the antenna, such as widening the working frequency band while reducing the size of the antenna. This paper summarizes the development process, current research, and the application of fractal tree antenna in miniaturization, multi-band and ultra-wide band antennas. And it also analyzes its development as well as application prospect.

Index Terms - fractal antenna, fractal tree, multi-band, ultrawide bandwidth, miniaturization
\end{abstract}

\section{Introduction}

In 1973, Benoit Mandelbrot first proposed the idea of fractal geometry and fractal dimension [1]. Then, fractal theory was applied in various fields of science research and engineering. In 1986, Kim and Jaggard first put forward that the fractal theory could be applied to the design of antenna arrays[2]. In 1996, C. Puente et al. studied the multiband properties of fractal tree nondeterministic antenna generated by electrochemical deposition [3]. In 2000, John P. Gianvittorio and Yahya Rahmat-Samii researched the miniaturization of dipole antennas that using fractal tree structures as end loads [4]. Since then, more and more researchers have been studying fractal tree antennas.

Fractal tree antenna has the common characteristics of fractal structure, namely space filling characteristic and selfsimilar characteristic. Fractal tree structure can improve the performance of the antenna, such as achieving miniaturization, multi-band or ultra wideband. The two dimensional (2-D) fractal tree dipole antennas have similar miniaturization effect, while the three dimensional (3-D) fractal trees have better miniaturization effect compared to the Koch dipole antennas [3]. The random fractal tree antennas generated by an electrochemical deposition process have denser multiband characteristic than the Sierpinski antenna [5]. Thus, it can be seen that fractal tree antennas have some unique advantages compared with other fractal antennas. An advantage of the fractal tree antenna is achieving flexibly reconfigurable antennas by adding reactive loads or RF switches in fractal tree structures [6]. So people pay special attention to the study of fractal tree antenna.

\section{The Current Research}

\section{A. Miniaturization}

Early, scholars mainly studied the miniaturization of the dipole antennas using fractal tree structures as end loads $[3,4$, 7,8]. In 2000 and 2002, the miniaturization of the two dimensional and three dimensional fractal tree dipole antennas was researched in $[3,4]$. The fractal tree structures are similar to Fig. 1 $(a, b)$. The dipole antennas using fractal tree structures as end loads have lower resonant frequency than a standard dipole with equal length, namely fractal tree structures achieve miniaturization effectively. In 2002, Joshua S. Petko and D. H. Werner improved the miniaturization of the 3-D fractal tree antennas by increasing the density of branches in [7]. It is found that the density of fractal tree antenna using fractal tree structures as end loads plays an important role in the designing of miniature antennas effectively. And increasing the density of branches can improve the miniaturization prominently. In 2004, Joshua S. Petko and D.H. Werner improved the antenna miniaturization by varying the elevation angle or the density of the branches in [8].The special Fibonacci number sequence which leads to variant branch length ratios can be utilized to design a novel fractal tree antenna in [9]. The novel fractal tree antenna gains better performance in the miniaturization, compared with the conventional designs. The methods mentioned above are just theoretical research. As the number of iterations increases, the complexity of the antennas grows rapidly. So these antennas occupying a certain space are not easy to be manufactured.

A fractal tree structure is utilized to design a dipole radio frequency identification (RFID) tag antenna on papery substrate $[10,11]$. The length of the fractal tree arm decreases approximately $18.4 \%$ compared to the half-wave dipole. The fractal tree antenna with simple structure is easier to process compared to the Manderlbrot structure in [10]. Reference $[12,13]$ propose a novel tree-shaped fractal patch antenna using arc branch of fractal tree structures as end loads. Geometry of the tree-shaped fractal dipole is similar to Fig. 1(c). The resonant frequency of the fractal antenna reduces approximately $37.5 \%$ compared to the zero iteration. It is found that the tree-shaped fractal structure achieves good performance in the miniaturization. A fractal tree antenna is designed using Rectangular, triangular and wired structure

\footnotetext{
* This work is supported by the director fund of State Key Laboratory of CEMEE (CEMEE2014Z0102B) and the National Natural Science Foundation of China (61001002)
} 
based on fractal tree geometry in [6]. H. Kimouche and $\mathrm{H}$. Zemmour designed a compact RFID tag antenna using fractal tree structure in [14], similar to Fig. 1 (d). The radiating element of the antenna consists of two radiating arms and one rectangular loop. The two radiating arms with fractal tree shape achieve the character of miniaturization and dual band, and the rectangular loop realizes an inductively coupled feeding. The electrical size of the antenna is $0.23 \lambda \times 0.091 \lambda$. It can be seen that the fractal tree structure can realize size reduction efficiently. In addition, the structure of the antenna is simple and easy to be manufactured.

\section{B. Reconfiguration}

Fractal tree structures are used to design reconfigurable antennas in $[6,15,16,17,18]$. In 2003, Joshua S. Petko and D.H. Werner designed two reconfigurable fractal tree antennas by introducing reactive loads and RF switches in the tree structure in [6]. In 2004, Joshua S. Petko and Douglas H. Werner introduced several design examples using reactive LC traps or RF switches in the tree structure to design miniature reconfigurable fractal tree antennas in [8]. And one reconfigurable antenna has an adjustable bandwidth reaching approximately $70 \%$ and realizes a $57 \%$ size reduction compared to the conventional half-wave dipole. The reconfigurable 2-D fractal tree antenna is designed by adding RF PIN diode switches in the tree structure to enhance MIMO channel capacity in [16]. A reconfigurable fractal tree antenna designed by using PIN diodes for multiband applications is proposed in [17]. The antenna realizes a tunable frequency ranging from $1.51 \mathrm{GHz}$ to $8.6 \mathrm{GHz}$. A reconfigurable fractal tree antenna for multi-band applications is designed by using PIN switches in [18]. The service bands of the antenna cover 2.4-2.48 GHz, 3.4-3.8GHz and other frequencies between $2.4 \mathrm{GHz}$ to $3.8 \mathrm{GHz}$.

\section{Multiband}

Because of self-similarity property, fractal tree structure is widely used to design multiband fractal antennas in $[3,19$, 20, 21, 22, 23, 24, 25, 14, 26, 27]. C. Puente et al. were the earliest researchers studying 2-D random fractal tree antenna created by an electrochemical deposition process in [3]. Hatem Rmili et al. studied the multiband behavior of a 3-D random fractal antenna in [19]. Compared to 2-D random fractal antenna, 3-D random fractal antenna has denser band distribution. As the random fractal antennas are generated randomly, the machining is complex and non-repeatable. The random fractal tree antennas generated by electrochemical deposition could have some difficulties in a practical application.

The multiband property of deterministic fractal tree antennas was studied in [20,21,22,23,24,25,14,26,27]. The multiband electromagnetic of a ternary tree-like fractal structure is studied in [20]. The tree-like structure is similar to Fig. 1(e). Reference [21] designed a three dimensional treelike fractal wire antenna working at L1-L2 GPS bands and WiFi. A fractal tree antenna for multiband applications, which is made based on ternary fractal tree geometry, is researched in
[22]. The fractal antenna works at the DVB-H, Bluetooth, WIMAX and WLAN. In 2009, Rui Guo et al. utilized the structure of the fractal binary tree to design a wide band antenna [23], working frequency ranging from $2.46 \mathrm{GHz}$ to $3.8 \mathrm{GHz}$. The parameters of the fractal antenna are optimized by using the genetic algorithm with the full-wave electromagnetic simulation and cluster parallel computation. It realizes antenna designing intelligently. In 2010, Aggarwal et al. designed a fractal patch antenna by exploiting the geometry of Pythagoras tree [24], which was similar to Fig. 1(f). The fractal antenna demonstrates great potential in multi-band applications. And its service bands contain WLAN $2.4 \mathrm{GHz}$ and WIMAX $3.5 \mathrm{GHz}$. A novel tree-shaped fractal antenna covering three operation bands, is designed in [24]. A dual band RFID tag antenna using third iteration fractal tree shape is designed in [14]. The tag antenna works at $915 \mathrm{MHz}$ as well as 2.4 GHz. In 2013, C. Varadhan et al. designed two kinds of tri-band RFID antennas by using fractal structures in [26]. One is a RFID reader antenna operating at $3.6 \mathrm{GHz}, 5.8 \mathrm{GHz}$, and $8.2 \mathrm{GHz}$; the other is a RFID tag antenna operating at $3.9 \mathrm{GHz}$, $5.9 \mathrm{GHz}$, and $8.2 \mathrm{GHz}$. The reader antenna with structure of modified fractal tree is designed. It can be seen that multiband characteristic can be realized effectively by using fractal tree structures. Reference [27] designs a dual-band RFID antenna for the dual-band RFID applications by placing a tree-like fractal structure between ground plane and the patch. The simple patch antenna, without the tree-like structure, has only single-band resonance with $7.2 \%$ bandwidth. The operating band is separated into $842 \mathrm{MHz}$ and $922 \mathrm{MHz}$ by applying the tree-like structure. Multiband antennas are successfully designed by combining fractal tree geometry with the practical applications in [14, 26, 27]. These fractal tree antennas have broad application prospects.

\section{Ultra-wide bandwidth}

The structure of fractal tree can also be exploited to design ultra-wideband antennas. A tree-like ultra-wide bandwidth (UWB) antenna is designed for the UWB applications in the MB-OFDM system in [28,29]. Through applying a fractal tree concept as well as partial ground plane technique, the antenna realizes impedance matching. In 2011, Javad Pourahmadazar et al. designed a modified UWB fractal tree planar antenna with a compact size in [30].Compared to T-Patch monopole antenna, the impedance bandwidth of first iteration antenna increases approximately $61.84 \%$. As the tree fractal iteration increases, the bandwidth of antenna can be broadened. The fifth iteration antenna covers frequency band from $2.64 \mathrm{GHz}$ to $11.14 \mathrm{GHz}(123.3 \%)$, with a compact size of $25 \times 25 \times 1 \mathrm{~mm}^{3}$. In 2012, M. Naser-Moghadasi et al. designed a novel UWB fractal tree-like antenna by using unit-cells of the fractal tree in [31], the tree structure is similar to Fig. 1(g). The third iteration antenna has a bandwidth of $2.1-11.52 \mathrm{GHz}(138 \%)$ with a compact dimension of $24 \times 24 \times 1 \mathrm{~mm} 3$.

As interference is a noticeable problem, anti-jamming signal is very important to UWB systems. To solve the interference problem, some scholars begin to research the 
UWB band-notched antennas. In 2013, M. Naser-Moghadasi et al. designed a compact fractal patch antenna with the notch band between 3.3-4.2 GHz in [32]. The folded T-shape element embedded in the fractal tree-shape radiating patch, realizes a band-rejection function to mitigate interference with existing $4 \mathrm{GHz}$ C-band system and WIMAX. A desired dual band-notch UWB antenna is designed in [33,34]. A narrowband fractal binary tree, etched into the radiating patch, achieves the dual band-notch functionality to suppress interference from UWB in WLAN bands.

\section{Conclusions}

We can design a compact multiband or UWB antenna by using fractal tree structure. This paper first introduces the development of fractal tree antenna, then expounded current state of the fractal tree research, and finally analyses the development and application prospect of fractal tree antennas. The fractal tree antenna can promote the development of fractal antenna engineering and accelerate the development of wireless communication. Although people have made some achievements in field of fractal tree antenna, we need to do further research. The fractal tree antenna may move forward into the following directions.

\section{A. compact multiband fractal antenna}

As fractal tree structure has space filling properties and self-similar characteristic, we can take advantage of the two properties to design compact multiband antennas. A compact fractal tree antenna may operate at RFID, mobile phones, WI-FI, Bluetooth, and WIMAX bands at the same time. And a small fractal tree antenna can realize all the applications in a smart-phone.

\section{B. A tunable or phase modulation fractal tree antenna}

The technique combining aspects of the theory of fractal tree geometry with RF MEMS switch technology can be used to design a reconfigurable antenna. It is more conducive to realize miniaturization, multi-band, and broadband by adding $\mathrm{RF}$ switches to fractal tree structures. The reconfigurable fractal tree antennas can be applied to MIMO antennas, SAR antennas, airborne antennas and so on.

\section{The UWB fractal tree antenna with the necessary band- notch functionality}

The interference between different communication systems is becoming more and more serious at present. To solve the interference problem, we need to design UWB antennas with the necessary band-notch by using fractal tree structures. The study on radiation theory and design method of UWB fractal tree antenna with the necessary band-notch functionality is urgently needed to suppress interference from UWB in WLAN, WIMAX, 4GHz C-band systems and so on.

\section{Combining fractal tree structures with other fractal structures.}

There are kinds of fractal antennas, and each of them has its own characteristics and advantages. As one kind of fractal antenna cannot meet the increasingly harsh requirements of communication systems, it is a promising development tendency in the future that to design a multi-functional antenna combining two or more fractal structures. The fractal antenna develops in diversify directions in the future. A fractal antenna with good performance can be designed by combining one fractal tree structure with others fractal structures.

\section{E. Fractal tree array antennas}

Compared with the traditional array antennas, the fractal array antennas have many advantages, such as multi-frequency and broadband characteristics, using iterative algorithms could achieve computing directional diagram rapidly and designing low side-lobe level antenna and so on. As the fractal tree array antenna belongs to fractal array antennas, so it has the benefits of fractal antenna array. And the application field of fractal tree antenna can be expanded by combining fractal tree technology with antenna array technology.

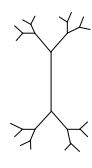

(a)

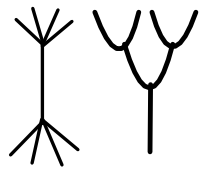

(b)

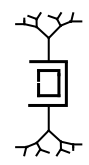

(d)

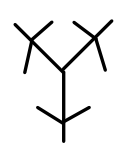

(e)

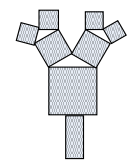

(

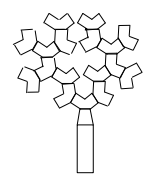

(g)
Fig. 1 different forms of fractal tree antenna

\section{References}

[1] B. B. Mandelbrot, "The fractal geometry of nature freeman," pp.224227, 1982

[2] Y. Kim and D. L. Jaggard, "The fractal random array," Proceedings of the IEEE, vol. 74, no. 9, pp. 1278-1280, 1986.

[3] C. Puente, J. Claret, F. Sagues, J. Romeu, M.Q.Lopez-Salvans and R. Pous, "Multiband properties of a fractal tree antenna generated by electrochemical deposition," Electronics Letters, vol.32, no.25, pp.22982299, December 1996.

[4] J. P. Gianvittorio and Y. Rahmat-Samii, "Fractal element antennas: a compilation of configurations with novel characteristics," in Proc. IEEE Antennas and Propagation Society Int. Symp., vol. 3, pp. 16881691, Salt Lake City, UT, July 2000.

[5] Douglas H.Werner and Suman Ganguly, "An overview of fractal antenna engineering research," IEEE Antennas and Propagation Magazine, vol. 45, no.2, pp. 38-57, February 2003.

[6] Joshua S.Petko and D.H. Werner ,"Reconfigurable miniature three dimensional fractal tree antennas," Antennas and Propagation Society International Symposium, vol.1, pp.371-374,2003.

[7] J. S. Petko and D. H.Werner, "Dense 3-D fractal tree structures as miniature end-loaded dipole antennas," in Proc. IEEE Antennas and Propagation Society Int. Symp., vol. 4, pp. 94-97,San Antonio, TX, June 2002.

[8] J. S. Petko and D. H. Werner, "Miniature reconfigurable threedimensional fractal tree antennas," IEEE Transactions on Antennas and Propagation, vol. 52, no.8, pp. 1945-1956, August 2004.

[9] Basak Ozbakis and Alp Kustepeli, "The resonant behavior of the fibonacci fractal tree antennas," Microwave and Optical Technology Letters, Vol. 50, No. 4, pp.1046-1050, April 2008.

[10] LAI Xiao-zheng, LIU Huan-bin, ZHAN Rui-nan, and LAI Sheng-li, "Papery substrate RFID tag antennas based on fractal geometry," Journal of Microwaves, vol.24, no.3, pp.37-39, June 2008.

[11] Yan Su, Xiao_zheng Lai and Sheng_li Lai, "Research on fractal tree RFID tag antenna on papery substrate," in Proceedings of the 4th International Conference on Wireless Communications, Networking and Mobile Computing, pp. 1-3, Dalian, October 2008. 
[12] Xianfeng Zeng, Chenxin Zhang, Yawei Wang and Cong Xie , "A novel planar tree-shaped fractal dipole patch antenna," Telecommunication Engineering,vol.50,no.5, PP. 76-79, May 2010.

[13] Xianfeng Zeng, Chenxin Zhang, Yawei Wang and Cong Xie, “ A novel planar tree-shaped fractal dipole patch antenna," in Proceedings of the International Conference on Microwave and Millimeter Wave Technology, pp.359-361, May 2010.

[14] H. Kimouche and H. Zemmour, "A compact fractal dipole antenna for $915 \mathrm{MHz}$ and $2.4 \mathrm{GHz}$ RFID tag applications," Progress In Electromagnetics Research Letters, vol. 26, pp.105-114, September 2011.

[15] Joshua S.Petko and D.H. Werner ,"Reconfigurable miniature three dimensional fractal tree antennas," Antennas and Propagation Society International Symposium, vol.1, pp. 371-374, 2003.

[16] Lackpour, A, Mookiah, P., Olivieri, M. and Dandekar, K, "Evaluation of the reconfigurable printed fractal tree antenna for enhanced pattern diversity in MIMO systems," Radio and Wireless Symposium , pp. 9699, New Orleans, LA, January 2010.

[17] Hüseyin Altun, Erdal Korkmaz, and Bahattin Türetken, "Reconfigurable fractal tree antenna for multiband applications," General Assembly and Scientific Symposium, 2011 XXXth URSI, pp. 1-4, Istanbul, August 2011

[18] Dr.V.R.Anitha and Malli yuva sindhu " "Simulation of a novel design fractal tree antenna for multiband applications with re-configurability," in Proceedings of the 2013 IEEE-APS Topical Conference on Antennas and Propagation in Wireless Communications, pp.148-1492, Torino, September 2013

[19] H. Rmili, O. Mrabet, J.-M. Floch and J.-L. Miane, "Study of an electrochemically deposited 3-D random fractal tree-monopole antenna," IEEE Transactions on Antennas and Propagation, vol. 55, no. 4, pp.1045-1050, April 2007.

[20] D.H. Werner, A. Rubio Bretones and B.R. Long, "Radiation characteristics of thin-wire ternary fractal trees," Electronics Letters, vol. 35, no. 8, pp. 609-610, April 1999.

[21] Renzo Azaro, Edoardo Zeni, Tommaso Gazzini, Ramona Dallapiccola and Andrea Massa, "Synthesis of a three-dimensional triband (L1-L2 GPS and Wi-Fi) prefractal tree antenna," Microwave and Optical Technology Letters, vol. 49,no. 9, PP. 2114-2118, September 2007.

[22] H.Kimouchee, M.Bitchikh, and B. Atrouz, "Novel design of a fractal monopole antenna for wireless communications," in Proceedings of the 14th Conference on Microwave Techniques, pp.1-5, Prague, April 2008
[23] Rui Guo, Xing Chen and Kama Huang, "A novel wideband microstrip fractal antenna based on the fractal binary tree," Electromagnetics, vol. 29, no. 4, pp. 283-290. May 2009.

[24] A. Aggarwal and M. V. Kartikeyan, "Pythagoras tree: A fractal patch antenna for multi-frequency and ultra-wide band-width operations," Progress in Electromagnetics Research C, vol. 16, PP. 25-35, 2010.

[25] Xianfeng Zeng, Chenxin Zhang, Cong Xie, Hongping An and Jiangang Liang, "Multi-band characteristic analysis of a novel tree-shaped fractal structure antenna," Telecommunication Engineering, vol.50, no.10, pp. 78-81, October 2010.

[26] Chitra Varadhan, Jayaram Kizhekke Pakkathillam, et al, "Triband antenna structures for RFID systems deploying fractal geometry," IEEE Antennas and Wireless Propagation Letters, vol. 12, PP.437-440, 2013.

[27] Guo Liu, Liang $\mathrm{Xu}$ and Zhensen $\mathrm{Wu}$, "Dual-Band microstrip RFID antenna with tree-Like fractal structure," IEEE Antennas and Wireless Propagation Letters, vol. 12, pp. 976-978, 2013.

[28] Jong K. park, Hee S. An and Jung N. Lee, "Design of the tree-shaped UWB antenna using fractal concept," Microwave and Optical Technology Letters, vol. 50, no. 1, pp. 144-150, January 2008.

[29] Hyo-Won Song, Hee-Soo An, et al, "Design of the tree-shaped UWB antenna using fractal concept," Microwave Conference, 2007. KJMW 2007, Korea-Japan, pp.73-76, November 2007.

[30] J. Pourahmadazar, C. Ghobadi , J. Nourinia and H. Shirzad, "Novel modified pythagorean tree fractal monopole antennas for UWB applications ," IEEE Antennas Wireless Propagation Letters, vol. 10, pp.484-487, 2011.

[31] M. Naser-Moghadasi, R. A. Sadeghzadeh, T. Aribi, T. Sedghi, and B.S. Virdee, "UWB monopole microstrip antenna using fractal tree unitcells," Microwave and Optical Technology Letters, vol. 54, no. 10, pp. 2366-2370, Oct.2012.

[32] M. Naser-Moghadasi, R. A. Sadeghzadeh, T. Aribi, T. Sedghi, and B.S. Virdee, "UWB CPW-Fed fractal patch antenna with band-notched function employing folded T-Shaped element," IEEE Antennas and Wireless Propagation Letters, vol. 12, pp.504-507, 2013.

[33] M.Naghshvarian Jahromi, A. Falahati, and R.M. Edwards, "Application of fractal binary tree slot to design and construct a dual band-notch CPW-ground-fed ultra-wide band antenna," IET Microwaves, Antennas \& Propagation, vol. 5, iss.12, pp. 1424-1430, 2011.

[34] A. Falahati, M. Naghshvarian-Jahromi, and R.M. Edwards, "Dual bandnotch CPW-Ground-Fed UWB antenna by fractal binary tree slot," in Proceedings of the Fifth International Conference on Wireless and Mobile Communications, pp. 385-390, Cannes, La Bocca, August 2009. 